\title{
Structure of solution sets to the nonlocal problems
}

\author{
Yi Cheng ${ }^{1 *}$, Ben Niu' and Cuiying $\mathrm{Li}^{2}$
}

"Correspondence: chengyi407@yahoo.com

${ }^{1}$ Department of Mathematics, Bohai University, Jinzhou, 121012, P.R. China

Full list of author information is available at the end of the article

\begin{abstract}
This paper deals with the structural properties of the solution set for a class of nonlinear evolution inclusions with nonlocal conditions. For the nonlocal problems with a convex-valued right-hand side it is proved that the solution set is compact $R_{\delta}$; it is the intersection of a decreasing sequence of nonempty compact absolute retracts. Then for the cases of a nonconvex-valued perturbation term it is proved that the solution set is path connected. Finally some examples of nonlinear parabolic problems are given.
\end{abstract}

MSC: 34B15; 34B16; 37J40

Keywords: evolution inclusions; nonlocal conditions; compact $R_{\delta} ;$ path connected

\section{Introduction}

In this paper, we study the structural properties of the solution set for a class of nonlinear evolution inclusions initiated in [1] with nonlocal conditions. The nonlocal Cauchy problems of evolution inclusions were investigated by Aizicovici and Lee [2], Aizicovici and McKibben [3], Aizicovici and Staicu [4], García-Falset [5], García-Falset and Reich [6], and Paicu and Vrabie [7], and by the references therein. However, as far as we know, not much work has been done for the topological structure of the solution set studied in this paper. In the past the topological structure of the solution set of differential inclusions in $\mathbb{R}^{N}$ has been investigated by Himmelberg and Van Vleck [8] and DeBlasi and Myjak [9]. Himmelberg and Van Vleck considered the topological structure of the solution set to the following differential inclusions:

$$
\dot{x}(t) \in F(t, x(t)), \quad x(0)=0,
$$

and they showed that the solution set was an $R_{\delta}$ set (see page 5 ). For the Cauchy problems the topological structure of the solution set of evolution inclusions was examined primarily by Papageorgiou and Shahzad [10], Andres and Pavlǎcková [11], Chen et al. [12] and Papageorgiou and Yannakakis [13] in a Banach space. For the optimization of this subject, we refer the reader to [14]. The corresponding work for multivalued evolution systems is limited to the Cauchy or periodic problems. The paper is largely motivated by the work of [1] in which the existence of solutions for first-order nonlinear evolution inclusions were proved. In [1], they only showed that there exists at least one solution for the cases of a

(c) 2016 Cheng et al. This article is distributed under the terms of the Creative Commons Attribution 4.0 International License (http://creativecommons.org/licenses/by/4.0/), which permits unrestricted use, distribution, and reproduction in any medium, provided you give appropriate credit to the original author(s) and the source, provide a link to the Creative Commons license, and indicate if changes were made. 
convex and of a nonconvex-valued perturbation term to the nonlocal problems, also prove that, under appropriate hypotheses, the extremal solution set is dense in the solution set of a system with a convexified right-hand side. However, it has to be noted that the topological structure of the solution set for this type of nonlinear evolution inclusions is an interesting problem which we intend to study in this paper.

In this paper we first of all prove that the solution set of nonlinear time-dependent evolution inclusions with a convex-valued right-hand side is compact $R_{\delta}$; it is the intersection of a decreasing sequence of nonempty compact absolute retracts in $C(I, H)$. Second, we go further and show that the solution set is path-connected in $C(I, H)$ for the case of a nonconvex-valued orientor field. Finally, some examples are also given to illustrate the effectiveness of our results. In particular, control systems given in this paper with a prior feedback, and systems with discontinuities, have a built-in multivalued character which is modeled appropriately by evolution inclusions.

\section{Preliminaries}

In this section we recall some basic definitions and facts from multivalued analysis, which will be needed later in this work. For further details we refer to the books of $\mathrm{Hu}$ and $\mathrm{Pa}$ pageorgiou [15, 16] and Zeidler [17]. Let $\mathcal{X}$ be a separable Banach space. The following notations are needed:

$$
\begin{aligned}
& P_{f}(\mathcal{X})=\{A \subset \mathcal{X}: A \text { is a nonempty; closed subset of } \mathcal{X}\}, \\
& P_{f c}(\mathcal{X})=\{A \subset \mathcal{X}: A \text { is a nonempty; closed and convex subset of } \mathcal{X}\} .
\end{aligned}
$$

Definition 2.1 A multifunction $H: \Omega \rightarrow P_{f}(\mathcal{X})$ is called 'measurable', if for all $y \in \mathcal{X}, \mathbb{R}_{+}$valued function $x \rightarrow d(y, H(x))=\inf \{\|y-v\|, v \in H(x)\}$ is measurable.

For given $A, B \in P_{f}(\mathcal{X})$, let

$$
d_{H}(A, B)=\max \left\{\sup _{a \in A} d(a, B), \sup _{b \in B} d(b, A)\right\} .
$$

The function $d_{H}: P_{f}(\mathcal{X}) \times P_{f}(\mathcal{X}) \rightarrow \mathbb{R}_{+}$is a metric on $P_{f}(\mathcal{X})$ and is called the 'Hausdorff metric'.

Definition 2.2 Let $Z$ be a separable Banach space, a multifunction $H: \mathcal{X} \rightarrow 2^{Z} \backslash \emptyset$ is called 'h-upper semicontinuous' (h-usc) if, for every $x \in \mathcal{X}$, the function $x^{\prime} \rightarrow d_{H}\left(H\left(x^{\prime}\right), H(x)\right)$ is continuous.

Let $Z$ be a complete metric space, also $H: \mathcal{X} \rightarrow P_{f}(Z)$ is called 'h-continuous' (resp. 'h-Lipschitz') if it is continuous (resp. Lipschitz) as a function from $Z$ into $P_{f}\left(Z, d_{H}\right)$. For details refer to [18].

Let $\left(V, H, V^{*}\right)$ be an evolution triple where the embedding $V \rightarrow H \rightarrow V^{*}$ is compact. Let $\langle\cdot, \cdot\rangle_{*}$ denote the pairing of an element $x \in V^{*}$ and an element $y \in V$. Let $\langle\cdot \cdot\rangle$ be the inner product on $H$, then $\langle\cdot, \cdot\rangle_{*}=\langle\cdot \cdot\rangle$, if $x, y \in H$. The dual space of $L^{p}(I, V)$ is $L^{q}\left(I, V^{*}\right)$ where $1<q \leq p<\infty, \frac{1}{p}+\frac{1}{q}=1$, and $I$ is an interval in $\mathbb{R}$. The norm in Banach space $L^{p}(I, V)$ will be denoted by $\|\cdot\|_{L^{p}(I, V)}$. Due to the reflexivity of $V$, both $L^{p}(I, V)$ and $L^{q}\left(I, V^{*}\right)$ are reflexive Banach spaces (see Zeidler [17], p.411). 
We define a Banach space $\mathcal{W}_{p q}(I)=\left\{x: x \in L^{p}(I, V), \dot{x} \in L^{q}\left(I, V^{*}\right)\right\}$ furnished with the norm $\|x\|_{\mathcal{W}}=\|x\|_{L^{p}(I, V)}+\|\dot{x}\|_{L^{q}\left(I, V^{*}\right)}$. The pairing between $L^{p}(I, V)$ and $L^{q}\left(I, V^{*}\right)$ is denoted by $\langle\cdot, \cdot\rangle_{* *}$.

By $S_{G}^{p}$ we denote the set of all $L^{p}(I, H)$-selectors of a multifunction $G$, i.e. $S_{G}^{p}=\{f \in$ $L^{p}(I, H): f(x) \in G(x)$ a.e. for $\left.x \in I\right\}$. We say that the set $S_{G}^{p}$ is decomposable if $\chi_{A} f_{1}+\chi_{A c} f_{2} \in$ $S_{G}^{p}$ where $\left(f_{1}, f_{2}, A\right) \in S_{G}^{p} \times S_{G}^{p} \times \Sigma$.

We recall some of the topological concepts which will be used to characterize the solution set of the evolution inclusion.

Definition 2.3 A subset $\mathcal{A} \subset \mathcal{X}$ is called 'path connected', if for every $x, y \subset \mathcal{A}$, there exists a path $h:[0,1] \rightarrow \mathcal{A}$ which joins $x$ to $y$.

For $\mathcal{A} \subset \mathcal{X}$ nonempty, we claim that $\mathcal{A}$ is a retract of $\mathcal{X}$, if there exists a continuous map $f: \mathcal{X} \rightarrow \mathcal{A}$ such that $\left.f\right|_{\mathcal{A}}=$ identity. It is clear to see that a retract $\mathcal{A} \subset \mathcal{X}$ is closed.

Definition 2.4 A closed subset $\mathcal{A}$ of $\mathcal{X}$ is called an absolute retract, if for any closed subset $C$ in every metric space $Y$, every continuous map $f: C \rightarrow \mathcal{A}$ can be extended to be a continuous function, $\widehat{f}: Y \rightarrow \mathcal{A}$.

Definition 2.5 A subset $\mathcal{A}$ of a metric space $\mathcal{X}$ is said to be contractible, if and only if there exist a continuous function $g:[0,1] \times \mathcal{A} \rightarrow \mathcal{X}$ and a point $a \in \mathcal{A}$ such that for all $x \in \mathcal{A}$ we have $g(0, x)=a$ and $g(1, x)=x$.

A subset $\mathcal{A}$ of a metric space $\mathcal{X}$ is said to be simply connected if every closed path in it is contractible to a point. A contractible set is both simply connected and path connected.

Definition 2.6 A subset $\mathcal{A}$ of a metric space is called an $R_{\delta}$ set if there exists a decreasing sequence $\left\{\mathcal{A}_{n}\right\}$ of compact and contractible sets such that

$$
\mathcal{A}=\bigcap_{n=1}^{\infty} \mathcal{A}_{n} .
$$

It is well known that any $R_{\delta}$ set is nonempty, compact, and connected. The following approximation result that we shall need can be proved as Proposition 4.1 of [9] with minor obvious modifications to accommodate the presence of $t \in I:=[0, T]$.

Lemma 2.1 If $F: I \times H \rightarrow P_{f c}\left(V^{*}\right)$ is measurable in $t$, $h$-usc in $x$, and $|F(t, x)| \leq \psi(t)$ for almost all $t \in I$ with $\psi(t) \in L^{q}(I)$, then there exists a sequence of multifunctions $F_{n}$ : $I \times H \rightarrow P_{f c}\left(V^{*}\right), n \geq 1$, such that for every $t \in I$ there exist $\mu(t)>0$ and $\epsilon>0$ such that if $x_{1}, x_{2} \in B_{\epsilon}(x)=\left\{y \in H:\|y-x\|_{H} \leq \epsilon\right\}$, then $d_{H}\left(F_{n}\left(t, x_{1}\right), F_{n}\left(t, x_{2}\right)\right) \leq \mu(t) \psi(t)\left\|x_{1}-x_{2}\right\|_{H}$ for almost all $t \in I$ (i.e., $F_{n}(t, x)$ is locally h-Lipschitz), $F(t, x) \subseteq \cdots \subseteq F_{n}(t, x) \subseteq F_{n+1}(t, x) \subseteq$ $\cdots,\left|F_{n}(t, x)\right| \leq \psi(t), n \in \mathbb{N}, F_{n}(t, x) \longrightarrow F(t, x)$ as $n \longrightarrow \infty$ for every $(t, x) \in I \times H$, and there exists $u_{n}: I \times H \rightarrow H$, measurable in $t$, locally Lipschitz in $x\left(\right.$ as $\left.F_{n}(t, x)\right)$ and $u_{n}(t, x) \in F_{n}(t, x)$ for every $(t, x) \in I \times H$. Moreover, if $F(t, \cdot)$ is $h$-continuous, then $t \rightarrow F_{n}(t, x)$ is measurable (hence $(t, x) \rightarrow F_{n}(t, x)$ is measurable too; see [19]). 


\section{Main results}

Let $I=[0, T]$ and $\left(V, H, V^{*}\right)$ be an evolution triple of spaces. Consider a nonlocal problems of evolution inclusions in the form

$$
\begin{aligned}
& \dot{x}(t)+B(t, x(t))+D x(t) \in \mathcal{F}(t, x(t)) \quad \text { a.e. for } t \in I, \\
& x(0)=\tau(x),
\end{aligned}
$$

where $B: I \times V \rightarrow V^{*}$ is a nonlinear operator, $D: V \rightarrow V^{*}$ is a bounded linear operator, $\tau: C(I, H) \rightarrow H$ is a continuous map, and $\mathcal{F}: I \times H \rightarrow 2^{V^{*}}$ is a multifunction to be given later. Some classical examples for $\tau$ are

(i) $\tau(x)=x(T)$;

(ii) $\tau(x)=-x(T)$;

(iii) $\tau(x)=\frac{1}{T} \int_{0}^{T} x(t) d t$;

(iv) $\tau(x)=\sum_{i=1}^{n} \theta_{i} x\left(t_{i}\right)$, where $0 \leq t_{1}<\cdots<t_{n} \leq T$ are arbitrary, but fixed and $\sum_{i=1}^{n}\left|\theta_{i}\right| \leq 1$.

We investigate the solutions of (3.1) in the weak sense. Let $u$ be a solution of problem (3.1), we mean a function $u \in W_{p q}(I)$ and there exists a function $f(t) \in \mathcal{F}(t, u)$ such that

$$
\langle\dot{u}(t), v\rangle_{*}+\langle B(t, u(t)), v\rangle_{*}+\langle D u(t), v\rangle_{*}=\langle f(t), v\rangle_{*^{\prime}}
$$

where $u(0)=\tau(u)$ for all $v \in V$ and almost all $t \in I$.

From [1], the existence of solution $x \in W_{p q}(I) \cap C(I, H)$ of problem (3.1) is obtained. Let $S$ denote the solution set of problem (3.1) defined as

$$
S=\left\{x \in W_{p q}(I) \cap C(I, H): x \text { is the solution of (3.1) }\right\} .
$$

In this paper, we can study the first structural property of the solution set $S \subset C(I, H)$ of (3.1). We show that $S$ is an $R_{\delta}$ set in $C(I, H)$.

We need the following hypotheses on the data of (3.1).

(H1) $B: I \times V \rightarrow V^{*}$ is an operator such that

(i) $t \rightarrow B(t, u)$ is measurable;

(ii) for almost all $t \in I$, there exists a constant $C_{1}>0$ such that

$$
\left\langle B\left(t, u_{1}\right)-B\left(t, u_{2}\right), u_{1}-u_{2}\right\rangle_{*} \geq C_{1}\left\|u_{1}-u_{2}\right\|_{H}^{p}
$$

for all $u_{1}, u_{2} \in V$, and the map $s \mapsto\langle B(t, u+s z), y\rangle_{*}$ is continuous on $[0,1]$ for all $u, y, z \in V$, and $p>1$;

(iii) there exist a constant $C_{2}>0$, a function $a(\cdot) \in L_{+}^{q}(I)$ where $q$ is the conjugate of $p>1$, and a nondecreasing continuous function $\alpha(\cdot)$ such that $\|B(t, u)\|_{V^{*}} \leq a(t)+C_{2} \alpha\left(\|u\|_{V}\right)$ for all $u \in V$, a.e. for $t \in I$;

(iv) there exist $C_{3}>0, C_{4}>0, b(\cdot) \in L^{1}(t)$ such that

$$
\begin{aligned}
\langle B(t, u), u\rangle_{*} \geq & C_{3}\|u\|_{V}^{p}-C_{4}\|u\|_{V}^{p-1} \\
& +\frac{1}{2 T}\|u(0)\|^{2}-b(t) \quad \text { a.e. } t \in I, \forall u \in V, 1<p \leq 2,
\end{aligned}
$$


and

$$
\langle B(t, u), u\rangle_{*} \geq C_{3}\|u\|_{V}^{p}-C_{4}\|u\|_{V}^{p-1}-b(t) \quad \text { a.e. } t \in I, \forall u \in V, p>2 .
$$

(i) $D: V \rightarrow V^{*}$ is a bounded linear self-adjoint operator such that $\langle D u, u\rangle_{*} \geq 0$, for all $u \in V$, for almost all $t \in I$;

(ii) there exists a continuous function $\tau: C(I, H) \rightarrow H$ such that

$$
\left\|\tau\left(u_{1}\right)-\tau\left(u_{2}\right)\right\|_{H} \leq\left\|u_{1}-u_{2}\right\|_{C(I, H)}, \quad \forall u_{1}, u_{2} \in C(I, H),
$$

and $\tau(0)=0$.

For every $f \in L^{q}(I, H)$, consider the following nonlinear evolution equation:

$$
\begin{aligned}
& \dot{u}(t)+B(t, u(t))+D u(t)=f(t), \quad \text { a.e. for } t \in I, \\
& u(0)=\tau(u)
\end{aligned}
$$

where $B: I \times V \rightarrow V^{*}$ is a nonlinear map, $D: V \rightarrow V^{*}$ is a bounded linear map, $\tau$ : $C(I, H) \rightarrow H$ is a continuous map. If hypotheses (H1), (H2) hold, then for every $f \in$ $L^{q}(I, H)$, from [1], the problem (3.2) has a unique solution $u \in W_{p q}(I) \subset C(I, H)$, moreover, $\|u\|_{C(I, H)} \leq M$, for some constant $M>0$. Let us define an operator $L: W_{p q}(I) \rightarrow L^{q}(I, H)$ as $L(u(t))=\dot{u}(t)+B(t, u(t))+D u(t)$ and $u(0)=\tau(u)$. By Theorem 3.1 of [1], then $L$ is oneto-one and surjective. Thus the inverse mapping $P: L^{q}(I, H) \rightarrow W_{p q}(I)$ is well defined. From (H1)(ii) and (H2), it is easy to deduce that $L: W_{p q}(I) \rightarrow L^{q}(I, H)$ is continuous. So $P: L^{q}(I, H) \rightarrow W_{p q}(I)$ is continuous. Because $W_{p q}(I)$ embeds continuously into $C(I, H)$ (see Proposition 23.23 of [17]), we see that $P: L^{q}(I, H) \rightarrow C(I, H)$ is continuous. Let $L^{q}(I, H)_{w}$ denote the Lebesgue-Bochner space furnished with the weak topology.

Let us give a proposition which is essential for our results.

Proposition 3.1 If hypotheses $(\mathrm{H} 1)$ and $(\mathrm{H} 2)$ hold, then $P: L^{q}(I, H)_{w} \rightarrow C(I, H)$ is sequentially continuous.

Proof Let

$$
W=\left\{v \in L^{q}(I, H):\|v(t)\|_{H} \leq \psi(t) \text { a.e. for } t \in I\right\}
$$

with $\psi(t) \in L_{+}^{q}(I)$. Similar to the step 2 of Theorem 3.1 in [1], we will show that there is a prior bound to problem (3.2) for each $f \in W$. Let $f_{n} \in W$ for every $n \geq 1$. From the definition of $W,\left\{f_{n}\right\}_{n \geq 1}$ is uniformly bounded in $L^{q}(I, H)$. By the Dunford-Pettis theorem, passing to a subsequence if necessary, we may assume that $f_{n} \rightarrow f$ weakly in $L^{q}(I, H)$ for some $f \in W$ as $n \longrightarrow \infty$. For the possible solution $u_{n}=P\left(f_{n}\right)$, we have

$$
\dot{u}_{n}(t)+B\left(t, u_{n}(t)\right)+D u_{n}(t)=f_{n}(t) \quad \text { a.e. for } t \in I \text {. }
$$

It follows that

$$
\left\langle\dot{u}_{n}, u_{n}\right\rangle_{* *}+\left\langle B\left(t, u_{n}\right), u_{n}\right\rangle_{* *}+\left\langle D u_{n}, u_{n}\right\rangle_{* *}=\left\langle f_{n}, u_{n}\right\rangle_{* *} .
$$


From (H1)(iv) and

$$
\begin{aligned}
& \int_{0}^{T}\left\langle\dot{u}_{n}(t), u_{n}(t)\right\rangle d t=\frac{1}{2}\left\|u_{n}(T)\right\|_{H}^{2}-\frac{1}{2}\left\|u_{n}(0)\right\|_{H}^{2}, \\
& \left\langle f_{n}, u_{n}\right\rangle_{* *} \leq\left\|f_{n}\right\|_{L^{q}\left(I, V^{*}\right)}\left\|u_{n}\right\|_{L^{p}(I, V)},
\end{aligned}
$$

we derive

$$
C_{3}\left\|u_{n}\right\|_{L^{p}(I, V)}^{p} \leq C_{4}\left\|u_{n}\right\|_{L^{p}(I, V)}^{p-1}+\left\|f_{n}\right\|_{L^{q}\left(I, V^{*}\right)}\left\|u_{n}\right\|_{L^{p}(I, V)}+\|b\|_{L^{1}},
$$

with $p>2$, and

$$
C_{3}\left\|u_{n}\right\|_{L^{p}(I, V)}^{p} \leq C_{4}\left\|u_{n}\right\|_{L^{p}(I, V)}^{p-1}+\left\|f_{n}\right\|_{L^{q}\left(I, V^{*}\right)}\left\|u_{n}\right\|_{L^{p}(I, V)}+\|b\|_{L^{1}}+\left\|u_{n}\right\|_{L^{p}(I, V)}^{2},
$$

with $1<p \leq 2$. Hence, we have, for all $n \in \mathbb{N},\left\|u_{n}\right\|_{L^{p}(I, V)} \leq M$ where $M>0$. From (3.2), it is easy to deduce that, for all $n \in \mathbb{N},\left\|u_{n}\right\|_{L^{q}\left(I, V^{*}\right)} \leq \bar{M}$ where $\bar{M}>0$. So we see that $\left\{u_{n}\right\}_{n \geq 1}$ is uniformly bounded in $W_{p q}(I)$. Due to the boundedness of the sequence $\left\{u_{n}\right\}_{n \geq 1} \subset W_{p q}(I)$, it follows that the sequence $\left\{u_{n}\right\}_{n \geq 1} \subset L^{q}\left(I, V^{*}\right)$ is uniformly bounded. So passing to a subsequence if necessary, we may assume that $\dot{u}_{n} \longrightarrow v$ weakly in $L^{q}\left(I, V^{*}\right)$. Obviously, $v=\dot{u}$ and $u_{n} \longrightarrow u$ weakly in $W_{p q}(I)$. Since the embedding $V \rightarrow H$ is compact, the embedding $W_{p q}(I) \rightarrow L^{p}(I, H)$ is compact. Thus, we have $u_{n} \rightarrow u$ in $L^{p}(I, H)$. Since the operator $B$ is hemicontinuous and monotone and $D$ is a continuous linear operator, we have $B\left(t, x_{n}\right) \longrightarrow B(t, x), D x_{n} \longrightarrow D x$ weakly in $L^{q}\left(I, V^{*}\right)$ as $n \longrightarrow \infty$. Therefore, we obtain $\dot{u}(t)+B(t, u(t))+D u(t)=f(t)$. Since the embedding $W_{p q}(I) \rightarrow C(I, H)$ is continuous, the sequence $\left\{u_{n}\right\}_{n \geq 1} \subset C(I, H)$ is uniformly bounded. Also, $\left\{u_{n}\right\}_{n \geq 1}$ is equicontinuous. By the Arzela-Ascoli theorem, passing to a subsequence, we may assume $u_{n} \longrightarrow u$ in $C(I, H)$. Since $\tau: C(I, H) \rightarrow H$ is continuous, we have $u_{n}(0)=\tau\left(u_{n}\right) \longrightarrow u(0)=\tau(u)$. Hence, $u=P(f)$. Because of $W_{p q}(I) \subset C(I, H)$, one has $u_{n}-u \in C(I, H)$. Note that

$$
\left\langle\dot{u}_{n}(t)-\dot{u}(t), u_{n}(t)-u(t)\right\rangle \leq\left\langle f_{n}(t)-f(t), u_{n}(t)-u(t)\right\rangle
$$

Integrating the above inequality over $[0, t]$, by hypotheses $(\mathrm{H} 1),(\mathrm{H} 2)$, for arbitrary $t \leq T$ we find

$$
\left\|u_{n}(t)-u(t)\right\|_{H}^{2} \leq 2 \int_{0}^{t}\left\langle f_{n}(s)-f(s), u_{n}(s)-u(s)\right\rangle d s+\left\|u_{n}(0)-u(0)\right\|_{H}^{2} .
$$

By

$$
\begin{aligned}
& \int_{0}^{t}\left\langle f_{n}(s)-f(s), u_{n}(s)-u(s)\right\rangle d s \\
& \quad \leq 2 \int_{0}^{T}\left\|f_{n}(t)\right\|_{H}\left\|u_{n}(t)-u(t)\right\|_{H} d t+2 \int_{0}^{T}\|f(t)\|_{H}\left\|u_{n}(t)-u(t)\right\|_{H} d t \\
& \quad \leq 4 \int_{0}^{T}|\psi(t)|\left\|u_{n}(t)-u(t)\right\|_{H} d t,
\end{aligned}
$$


it follows that

$$
\begin{aligned}
\left\|u_{n}(t)-u(t)\right\|_{H}^{2} & \leq 4 \int_{0}^{T}|\psi(t)|\left\|u_{n}(t)-u(t)\right\|_{H} d t+\left\|u_{n}(0)-u(0)\right\|_{H}^{2} \\
& \leq 4 \int_{0}^{T}|\psi(t)|\left\|u_{n}(t)-u(t)\right\|_{H} d t+\left\|\tau\left(u_{n}\right)-\tau(u)\right\|_{H}^{2} \\
& \leq 4\|\psi\|_{L^{q}}\left\|u_{n}-u\right\|_{L^{p}(I, H)}+\left\|\tau\left(u_{n}\right)-\tau(u)\right\|_{H}^{2} \\
& \longrightarrow 0
\end{aligned}
$$

as $n \longrightarrow \infty$.

Then we get

$$
\max _{t \in I}\left\|u_{n}(t)-u(t)\right\|_{H} \longrightarrow 0 \quad \text { as } n \longrightarrow \infty
$$

So, $u_{n} \longrightarrow u$ in $C(I, H)$, i.e. $P: L^{q}(I, H)_{w} \longrightarrow C(I, H)$ is sequentially continuous.

To prove such a structural result for the solution set of (3.1) we need the following hypotheses on $\mathcal{F}(t, x)$ :

(H3) $\mathcal{F}: I \times H \rightarrow 2^{H}$ is a multifunction with closed and convex values such that

(i) $(t, u) \rightarrow \mathcal{F}(t, u)$ is graph measurable;

(ii) for almost all $t \in I, u \rightarrow \mathcal{F}(t, u)$ has a closed graph;

(iii) there exist a function $b_{1}(\cdot) \in L_{+}^{q}(I)$ and a constant $C_{5}>0$ such that

$$
\begin{aligned}
|\mathcal{F}(t, u)| & =\sup \left\{\|f\|_{H}: f \in \mathcal{F}(t, u)\right\} \\
& \leq b_{1}(t)+C_{5}\|u\|_{H}^{k-1}, \quad \forall x \in H, \text { a.e. for } t \in I,
\end{aligned}
$$

where $1 \leq k<p$.

Theorem 3.1 Under assumptions (H1)-(H3), $S$ is an $R_{\delta}$ set in $C(I, H)$.

Proof From the prior estimation executed in the proof of Theorem 3.1 in [1], without loss of generality, we may suppose that, for almost all $t \in I$, all $u \in H$, and all $h(t) \in \mathcal{F}(t, u)$, we have $\|h(t)\|_{H} \leq \psi(t)$ where $\psi \in L_{+}^{q}(I)$. Applying Lemma 2.1, we can obtain a sequence of multifunctions $\mathcal{F}_{n}: I \times H \rightarrow P_{f c}(H)$. For every $n \geq 1$, consider the following evolution inclusion:

$$
\begin{aligned}
& \dot{u}(t)+B(t, u(t))+D u(t) \in \mathcal{F}_{n}(t, u(t)), \quad \text { a.e. for } t \in I, \\
& u(0)=\tau(u) .
\end{aligned}
$$

From Theorem 4.1 of [1], we see that the solution set $S_{n} \subseteq W_{p q}(I) \subset C(I, H)$ of problem (3.4) is compact in $C(I, H)$. The following two steps are needed to complete the proof.

Step 1 . This set $S_{n}$ is contractible.

Let $w_{n}(t, u)$ be measurable selector of $\mathcal{F}_{n}(t, u)$ generated by Lemma 2.1 , the locally Lipschitz continuous in $u \in H$. Fixing $w_{n}(t, u) \in \mathcal{F}_{n}(t, u)$, let $\widehat{u}=P\left(w_{n}(t, u)\right) \in S_{n}$, where $P$ is 
defined as before. Consider the following Cauchy problem:

$$
\begin{aligned}
& \dot{z}(t)+B(t, z(t))+D z(t)=w_{n}(t, z) \quad \text { a.e. for } t \in[\delta T, T], \\
& z(\delta T)=\delta u(\delta T)+(1-\delta) \widehat{u}(0) .
\end{aligned}
$$

It is easy to check the existence of solution in (3.5) (see [20]). Because $B$ is hemicontinuous and monotone, $D$ is a continuous linear operator and $w_{n}(t, u)$ be the locally Lipschitz continuous in $u \in H$, the solution of (3.5) is unique. For every $\delta \in[0,1]$ and every $u \in S_{n}$, let $z(\delta, u)(t) \in W_{p q}(I)$ be the unique solution of (3.5). In order for this set $S_{n}$ to be contractible, for given $\widehat{u} \in S_{n}$, we only need to find a continuous function $\mu:[0,1] \times S_{n} \rightarrow S_{n}$ such that, for all $u \in S_{n}$, we have $\mu(0, u)(t)=\widehat{u}(t)$ and $\mu(1, u)(t)=u(t)$. So we can define $\mu:[0,1] \times S_{n} \rightarrow S_{n}$ by

$$
\mu(\delta, u)(t)=\left(\begin{array}{cc}
\delta u(t)+(1-\delta) \widehat{u}(0) & \text { for } t \in[0, \delta T], \\
z(\delta, u)(t) & \text { for } t \in(\delta T, T]
\end{array}\right)
$$

If $\delta=0$, by $(\mathrm{H} 1)$ and $(\mathrm{H} 2)$, we know that $z(t)=\widehat{u}(t)$ for every $t \in I$. So $\mu(0, u)(t)=\widehat{u}$. From the definition of $\mu(\delta, u)(t)$, obviously, $\mu(1, u)(t)=u(t)$ for every $u(t) \in S_{n}$. We show that $\mu(\delta, u)(t) \in S_{n}$ for each $(\delta, u) \in[0,1] \times S_{n}$. Note that for each $u \in S_{n}$, there exists $\tilde{f} \in \mathcal{F}_{n}(t, u)$, such that $u=P(\widetilde{f})$. Let

$$
v(t)=\tilde{f} \chi_{[0, \delta T]}(t)+w_{n} \chi_{(\delta T, T]}(t) \quad \text { for } t \in I,
$$

where $\chi$ is the characteristic function. Since $\mathcal{F}_{n}(t, u)$ is closed and convex-valued, from the definition of a decomposable value (see Section 2), we see that $\mathcal{F}_{n}(t, u)$ is decomposable, from which one deduces $v \in \mathcal{F}_{n}(t, u)$. Obviously, $P(v)=u(t)$ for all $t \in[0, \delta T]$ and $P(v)=$ $z(\delta, u)(t)$ for all $t \in(\delta T, T]$, which means $P(v)=\mu(\delta, u)$ for any $\delta \in[0,1]$. Hence, $\mu(\delta, u) \in$ $S_{n}$.

To prove that $S_{n}$ is contractible, we first note that $\mu:[0,1] \times S_{n} \rightarrow S_{n}$ and $\mu(0, u)(t)=\widehat{u}(t)$ and $\mu(1, u)(t)=u(t)$ for every $u \in S_{n}$. Next, it remains to show that $\mu(\delta, u)(t)$ is continuous in $[0,1] \times C(I, H)$. To this aim, let $\left(\delta_{m}, u_{m}\right) \longrightarrow(\delta, u)$ in $[0,1] \times S_{n}$. Next, we will distinguish two distinct cases to proceed.

$I: \delta_{m} \geq \delta$ for every $m \geq 1$. Let $v_{m}(t)=\mu\left(\delta_{m}, u_{m}\right)(t)$, for $t \in I$. Obviously, $v_{m} \in S_{n}, m \geq 1$. Due to the compactness of the solution set $S_{n}$ in $C(I, H)$, by passing to a subsequence if necessary, we may suppose that $v_{m} \longrightarrow v$ in $C(I, H)$ as $m \longrightarrow \infty$. Next, we only need to check $v(t)=\mu(\delta, u)(t)$. Clearly, $[0, \delta T] \subseteq\left[0, \delta_{m} T\right]$, by the definition of $\mu(\delta, u)$, one has $v_{m}(t)=\delta_{m} u_{m}(t)+\left(1-\delta_{m}\right) \widehat{u}(0)$ for $0 \leq t \leq \delta_{m} T$. As $\left(\delta_{m}, u_{m}\right) \rightarrow(\delta, u)$ in $[0,1] \times C(I, H)$, we obtain $v(t)=\delta u(t)+(1-\delta) \widehat{u}(0)$ for $0 \leq t \leq \delta T$. Also let $y \in W_{p q}(I)$ be the unique solution of the following equation:

$$
\begin{aligned}
& \dot{y}(t)+B(t, y(t))+D y(t)=w_{n}(t, v) \quad \text { a.e. for } t \in[\delta T, T], \\
& y(\delta T)=v(\delta T) .
\end{aligned}
$$

Let $m \geq N$ be large enough for some constant $N>0$, by $(3.6), v_{m}(\cdot)$ satisfies

$$
\dot{v}_{m}(t)+B\left(t, v_{m}(t)\right)+D v_{m}(t)=w_{n}\left(t, v_{m}(t)\right) \quad \text { a.e. for } t \in\left[\delta_{m} T, T\right] .
$$


Exploiting the monotonicity of the operator $B(t, \cdot)$ and $D$, and combining (3.6) with (3.7), we find

$$
\begin{aligned}
& \left\langle\dot{y}(t)-\dot{v}_{m}(t), y(t)-v_{m}(t)\right\rangle \\
& \quad \leq\left\langle w_{n}(t, v(t))-w_{n}\left(t, v_{m}(t)\right), y(t)-v_{m}(t)\right\rangle \quad \text { a.e. for } t \in\left[\delta_{N} T, T\right] .
\end{aligned}
$$

Taking the integral over the above inequality from $\delta_{N} T$ to $t(t \leq T)$, we have

$$
\begin{aligned}
& \left\|y(t)-v_{m}(t)\right\|_{H}^{2} \\
& \quad \leq\left\|y\left(\delta_{N} T\right)-v_{m}\left(\delta_{N} T\right)\right\|_{H}^{2}+2 \int_{\delta_{N} T}^{t}\left\|w_{n}(s, v(s))-w_{n}\left(s, v_{m}(s)\right)\right\|_{H}\left\|y(s)-v_{m}(s)\right\|_{H} d s .
\end{aligned}
$$

By Brezis [21], p.157, we obtain

$$
\left\|y(t)-v_{m}(t)\right\|_{H} \leq\left\|y\left(\delta_{N} T\right)-v_{m}\left(\delta_{N} T\right)\right\|_{H}+\int_{\delta_{N} T}^{t}\left\|u_{n}(s, v(s))-u_{n}\left(s, v_{m}(s)\right)\right\|_{H} d s
$$

Passing to the limit as $m \longrightarrow \infty$, and recalling that $v_{m} \longrightarrow v$ in $C(I, H)$ and $w_{n}(t, u)$ is locally Lipschitz continuous in $u$, we get $\|y(t)-v(t)\|_{H} \leq\left\|y\left(\delta_{N} T\right)-v\left(\delta_{N} T\right)\right\|_{H}$ for $\delta_{N} T \leq t \leq T$. Since $y(t)$ is the solution in (3.6), $y \in C(I, H)$. So $y(t)$ is continuous with respect to $t$. Finally, $y\left(\delta_{N} T\right) \rightarrow v(\delta T)$ and $v\left(\delta_{N} T\right) \rightarrow v(\delta T)$ in $H$ as $N \rightarrow \infty$, in the limit we get $y(t)=v(t)$ for every $\delta T \leq t \leq T$. So

$$
\begin{aligned}
& \dot{v}(t)+B(t, v(t))+D v(t)=w_{n}(t, v(t)) \quad \text { a.e. for } t \in[\delta T, T], \\
& v(\delta T)=\delta u(\delta T)+(1-\delta) \widehat{u}(0),
\end{aligned}
$$

hence $v=\mu(\delta, u)$. Therefore $\mu\left(\delta_{m}, u_{m}\right) \longrightarrow \mu(\delta, u)$ in $C(I, H)$ as $\left(\delta_{m}, u_{m}\right) \longrightarrow(\delta, u)$.

II: $\delta_{m} \leq \delta$ for every $m \geq 1$. Keeping the notation as in case I, we have $\left[0, \delta_{m} T\right] \subseteq[0, \delta T]$. By the definition of $\mu(\delta, u)$, one has $v_{m}(t)=\delta_{m} u_{m}(t)+\left(1-\delta_{m}\right) \widehat{u}(0)$ for $0 \leq t \leq \delta_{m} T$. As $\left(\delta_{m}, u_{m}\right) \longrightarrow(\delta, u)$ in $[0,1] \times C(I, H)$, we get $v(t)=\delta u(t)+(1-\delta) \widehat{u}(0)$ for $0 \leq t \leq \delta T$. Also let $y \in W_{p q}(I)$ be the unique solution of the following equation:

$$
\begin{aligned}
& \dot{y}(t)+B(t, y(t))+D y(t)=w_{n}(t, v) \quad \text { a.e. for } t \in[\delta T, T], \\
& y(\delta T)=v(\delta T) .
\end{aligned}
$$

Let $m \geq N$ large enough for some constant $N>0$, by $(3.8), v_{m}(\cdot)$ satisfies

$$
\dot{v}_{m}(t)+B\left(t, v_{m}(t)\right)+D v_{m}(t)=w_{n}\left(t, v_{m}(t)\right) \quad \text { a.e. for } t \in\left[\delta_{N} T, T\right] .
$$

Due to the monotonicity of the operator $B(t, \cdot)$ and $D$, and combining (3.8) with (3.9), we have

$$
\begin{aligned}
& \left\langle\dot{y}(t)-\dot{v}_{m}(t), y(t)-v_{m}(t)\right\rangle \\
& \quad \leq\left\langle w_{n}(t, v(t))-w_{n}\left(t, v_{m}(t)\right), y(t)-v_{m}(t)\right\rangle \quad \text { a.e. for } t \in[\delta T, T] .
\end{aligned}
$$


Integrating the above inequality over $[\delta T, t), t \leq T$, we have

$$
\begin{aligned}
& \left\|y(t)-v_{m}(t)\right\|_{H}^{2} \\
& \quad \leq\left\|y(\delta T)-v_{m}(\delta T)\right\|_{H}^{2}+2 \int_{\delta T}^{t}\left\|w_{n}(s, v(s))-w_{n}\left(s, v_{m}(s)\right)\right\|_{H}\left\|y(s)-v_{m}(s)\right\|_{H} d s .
\end{aligned}
$$

As before, we obtain

$$
\left\|y(t)-v_{m}(t)\right\|_{H} \leq\left\|y(\delta T)-v_{m}(\delta T)\right\|_{H}+\int_{\delta T}^{t}\left\|w_{n}(s, v(s))-w_{n}\left(s, v_{m}(s)\right)\right\|_{H} d s .
$$

Taking the limit as $m \longrightarrow \infty$, and recalling that $v_{m} \longrightarrow v$ in $C(I, H)$ and $w_{n}(t, x)$ is the locally Lipschitz continuous in $x \in H$, we get $\|y(t)-v(t)\|_{H} \leq\|y(\delta T)-v(\delta T)\|_{H}$ for $\delta T \leq t \leq T$. Since $y(t)$ is the solution in (3.8), $y(\delta T)=v(\delta T)$. Finally, in the limit we get $y(t)=v(t)$ for every $\delta T \leq t \leq T$. So

$$
\begin{aligned}
& \dot{v}(t)+B(t, v(t))+D v(t)=w_{n}(t, v(t)) \quad \text { a.e. for } t \in[\delta T, T], \\
& v(\delta T)=\delta u(\delta T)+(1-\delta) \widehat{u}(0),
\end{aligned}
$$

hence $v=\mu(\delta, u)$. Therefore $\mu\left(\delta_{m}, u_{m}\right) \longrightarrow \mu(\delta, u)$ in $C(I, H)$ as $\left(\delta_{m}, u_{m}\right) \longrightarrow(\delta, u)$.

In fact, we can always get a subsequence of $\left\{\delta_{m}\right\}_{m \geq 1}$ conforming to I or II. Thus we have established the continuity of $\mu(\delta, u)$. Therefore, for every $n \geq 1, S_{n} \subseteq C(I, H)$ is compact and contractible.

Step 2. $S=\bigcap_{n \geq 1} S_{n}$.

Evidently, $S \subseteq \bigcap_{n \geq 1} S_{n}$. Let $u \in \bigcap_{n \geq 1} S_{n}$. Then through definition $u=P\left(f_{n}\right)$, where $f_{n} \in$ $S_{\mathcal{F}_{n}\left(\cdot, u_{n}(\cdot)\right)}^{q}$ (the set of all $L^{q}(I, H)$-selectors of $\left.\mathcal{F}_{n}\right), n \geq 1$. By passing to a subsequence if necessary, we may assume that $f_{n} \longrightarrow f$ weakly in $L^{q}(I, H)$. Then $f \in S_{\mathcal{F}(\cdot, u(\cdot))}^{q}$ (see Theorem 3.2 of [1]). So $u=P(f)$ with $f \in S_{\mathcal{F}(\cdot, u(\cdot))}^{q}$ from which we conclude that $S=\bigcap_{n \geq 1} S_{n}$. Finally, by Steps 1 and 2, Hyman's result [22] implies that $S$ is an $R_{\delta}$ set in $C(I, H)$.

An immediate conclusion of Theorem 3.1 is the following result for the multivalued problem (3.1).

Remark 3.1 Assume (H1)-(H3), then for every $t \in I, S(t)=\{x(t) \mid x \in S\}$ (the reachable set at time $t \in I)$ is compact and connected in $H$.

We can establish an analogous result for the topological structure of a nonconvex evolution inclusion (i.e., $\mathcal{F}(t, u)$ has nonconvex values) provided we strengthen our assumption on the continuity of $\mathcal{F}(t, u)$. In fact, in this case we can see that the solution set is path connected.

To prove such a structural result for the solution set of (3.1) we need the following hypotheses on $\mathcal{F}(t, x)$ and $\tau$ :

$\mathcal{F}: I \times H \rightarrow P_{f}(H)$ is a multifunction such that

(i) $t \rightarrow \mathcal{F}(t, u)$ is measurable;

(ii) for every $u_{1}, u_{2} \in H, h\left(\mathcal{F}\left(t, u_{1}\right), \mathcal{F}\left(t, u_{2}\right)\right) \leq z(t)\left\|u_{1}-u_{2}\right\|_{H}$ a.e. with $z(t) \in L_{+}^{q}(I)$ 
(iii) there exist a function $b_{2}(\cdot) \in L_{+}^{q}(I)$ and a constant $C_{5}>0$ such that

$$
|\mathcal{F}(t, u)|=\sup \left\{\|f\|_{H}: f \in \mathcal{F}(t, u)\right\} \leq b_{2}(t)+C_{5}\|u\|_{H}^{k-1} \quad \forall u \in V \text { a.e. for } t \in I
$$

with $1 \leq k<p$.

$(\mathrm{H} 2)_{1}$

(i) $D: V \rightarrow V^{*}$ is a bounded linear self-adjoint operator such that $\langle D u, u\rangle_{*} \geq 0$, for all $u \in V$, a.e. for $t \in I$;

(ii) there exists a continuous function $\tau: C(I, H) \rightarrow H$ such that

$$
\left\|\tau\left(u_{1}\right)-\tau\left(u_{2}\right)\right\|_{H} \leq \mathcal{L}\left\|u_{1}-u_{2}\right\|_{C(I, H)}, \quad \forall u_{1}, u_{2} \in C(I, H)
$$

where $\mathcal{L} \in[0,1), \tau(0)=0$.

Theorem 3.2 If hypotheses (H1), $(\mathrm{H} 2)_{1}$, and ( $\left.\mathrm{H} 4\right)$ are satisfied, then $S \subseteq C(I, H)$ is nonempty and path connected.

Proof The prior estimation conducted in the three part of the proof of Theorem 3.1 in [1] is still valid here. So according to it, we see that without any loss of generality we may suppose that $|\mathcal{F}(t, u)| \leq \psi(t)$ a.e. with $\psi(t) \in L_{+}^{q}(I)$. Let $W_{\alpha}=\left\{g \in L^{q}(I, H)\right.$ : $\|g(t)\|_{H} \leq \psi(t)$ a.e. for $\left.t \in I\right\}$, and the multifunction $N: W_{\alpha} \rightarrow P_{f}\left(L^{q}(I, H)\right)$ be defined by $N(g)=S_{\mathcal{F}(, P(g)(\cdot))}^{q}\left(\right.$ here $P(g)$ is defined as before). Next, we present a new norm on $L^{q}(I, H)$ defined by

$$
\|g\|_{q}=\left(\int_{0}^{T} \exp ^{-r \int_{0}^{t} z^{q}(s) d s}\|g(t)\|_{H}^{q} d t\right)^{\frac{1}{q}}
$$

where $r$ is a number satisfying $r>T^{\frac{q}{p}}, p>1$, and $q$ is the conjugate of $p$. It is easy to check that the norm $\|\cdot\|_{q}$ is the equivalent of $\|\cdot\|_{L^{q}(I, H)}$. Denote by $d_{q}(\cdot, \cdot)$ and $d_{\widehat{H}}(\cdot, \cdot)$ the distance function and Hausdorff metric, respectively, generated by $\|\cdot\|_{q}$. Let $f, g \in W_{\alpha}$ and $v \in N(g)$. Let $\epsilon>0$ and define

$$
D_{\epsilon}(t)=\left\{w \in \mathcal{F}(t, P(f)):\|v(t)-w(t)\|_{H} \leq d(v(t), F(t, P(f)(t)))+\epsilon\right\} .
$$

Let $b(t, w)=d(v(t), \mathcal{F}(t, P(f)))-\|v-w\|_{H}+\epsilon$. Obviously, for every $t \in I, D_{\epsilon}(t) \neq \emptyset$ and $\mathbf{G r} D_{\epsilon}=\{(t, w) \in \mathbf{G r} \mathcal{F}(t, P(f)(t)): b(t, w) \geq 0\}$. In fact, because of (H4)(i) and (ii), $(t, u) \rightarrow \mathcal{F}(t, u)$ is measurable, thus $t \rightarrow \mathcal{F}(t, P(f)(t))$ is measurable. So, $(t, w) \rightarrow b(t, w)$ is a Carathéodory function, thus jointly measurable. Hence $\operatorname{Gr} D_{\epsilon} \in \mathcal{B}(I) \times \mathcal{B}(H)$. Apply Aumann's selection theorem to get $w: I \rightarrow H$ measurable such that $w(t) \in D_{\epsilon}(t)$ a.e. for $t \in I$. Then we find

$$
\begin{aligned}
d_{q}(v, N(f)) & \leq\|v-w\|_{q} \\
& =\left(\int_{0}^{T}\|v-w\|_{H}^{q} \exp ^{-r \int_{0}^{t} z^{q}(s) d s} d t\right)^{\frac{1}{q}} \\
& \leq\left(\int_{0}^{T}[d(v(t), \mathcal{F}(t, P(f)(t)))]^{q} \exp ^{-r \int_{0}^{t} z^{q}(s) d s} d t\right)^{\frac{1}{q}}+(T)^{\frac{1}{q}} \epsilon
\end{aligned}
$$




$$
\begin{aligned}
& \leq\left(\int_{0}^{T}[h(\mathcal{F}(t, P(g)(t)), \mathcal{F}(t, P(f)(t)))]^{q} \exp ^{-r \int_{0}^{t} z^{q}(s) d s} d t\right)^{\frac{1}{q}}+(T)^{\frac{1}{q}} \epsilon \\
& \leq\left(\int_{0}^{T} z^{q}(t)\|P(f)(t)-P(g)(t)\|_{H}^{q} \exp ^{-r \int_{0}^{t} z^{q}(s) d s} d t\right)^{\frac{1}{q}}+(T)^{\frac{1}{q}} \epsilon .
\end{aligned}
$$

For every $y \in C(I, H)$, consider the following differential inclusion:

$$
\begin{aligned}
& \dot{x}(t)+B(t, x(t))+D x(t)=f(t), \quad \text { a.e. for } t \in I, \\
& x(0)=\tau(y),
\end{aligned}
$$

where $f(t) \in \mathcal{F}(t, x(t))$. Let $S(y)$ denote the solution set of (3.11), by Theorem 3.1 in [1], we have $S(y) \neq \emptyset$. Since $B(t, \cdot)$ is monotone, $D$ is a continuous linear map, and $P(f)(0)=P(g)(0)$ $(P(f), P(g) \in S(y))$, we have

$$
\frac{1}{2}\|P(f)(t)-P(g)(t)\|_{H}^{2} \leq \int_{0}^{t}\|P(f)-P(g)\|_{H}\|f(s)-g(s)\|_{H} d s .
$$

Hence, we obtain $\|P(f)(t)-P(g)(t)\|_{H} \leq \int_{0}^{t}\|f(s)-g(s)\|_{H} d s$ for all $t \in I$ (see Brezis [21], p.157), then $\|P(f)(t)-P(g)(t)\|_{H}^{q} \leq T^{\frac{q}{p}} \int_{0}^{t}\|f(s)-g(s)\|_{H}^{q} d s$. From (3.10), it follows that

$$
\begin{aligned}
d_{q}(v, N(f)) & \leq\left(T^{\frac{q}{p}} \int_{0}^{T} z^{q}(t) \int_{0}^{t}\|f(s)-g(s)\|_{H}^{q} d s \exp ^{-r} \int_{0}^{t} z^{q}(s) d s d t\right)^{\frac{1}{q}}+(T)^{\frac{1}{q}} \epsilon \\
& \leq\left(-\frac{1}{r} T^{\frac{q}{p}} \int_{0}^{T} \int_{0}^{t}\|f(s)-g(s)\|_{H}^{q} d s \frac{d}{d t}\left(\exp ^{-r} \int_{0}^{t} z^{q}(s) d s\right) d t\right)^{\frac{1}{q}}+(T)^{\frac{1}{q}} \epsilon \\
& \leq\left(\frac{1}{r} T^{\frac{q}{p}} \int_{0}^{T} \exp ^{-r} \int_{0}^{t} z^{q}(s) d s\|f(t)-g(t)\|_{H}^{q} d t\right)^{\frac{1}{q}}+(T)^{\frac{1}{q}} \epsilon \\
& \leq\left(\frac{1}{r}\right)^{\frac{1}{q}} T^{\frac{1}{p}}\|f(t)-g(t)\|_{q}+(T)^{\frac{1}{q}} \epsilon .
\end{aligned}
$$

Let $\epsilon \rightarrow 0$ to get $d_{q}(v, N(f)) \leq\left(\frac{1}{r}\right)^{\frac{1}{q}} T^{\frac{1}{p}}\|f(t)-g(t)\|_{q}$. Exchanging the roles of $f$ and $g$ we also get $d_{q}(u, N(g)) \leq\left(\frac{1}{r}\right)^{\frac{1}{q}} T^{\frac{1}{p}}\|f(t)-g(t)\|_{q}$. Thus, finally, $d_{\widehat{H}}(N(f)(t), N(g)(t)) \leq\left(\frac{1}{r}\right)^{\frac{1}{q}} T^{\frac{1}{p}} \| f(t)-$ $g(t) \|_{q}$ where $\left(\frac{1}{r}\right)^{\frac{1}{q}} T^{\frac{1}{p}}<1$. Set $\Theta=\left\{g \in W_{\alpha}: g \in N(g)\right\}$. From Nadler's fixed-point theorem [23] we get $\Theta \neq \emptyset$, and then from Theorem 1 in [24] we see that $\Theta$ is an absolute retract in $L^{q}(I, H)$. Since an absolute retract is path connected, we see that $\Theta$ is path connected. Then $P(\Theta)$ is path connected in $C(I, H)$. However, note that $P(\Theta)=S(y)$, to conclude that $S(y)$ is nonempty and path connected in $C(I, H)$. For every $y_{1}, y_{2} \in C(I, H)$, we see that there exist $x_{1}(t) \in S\left(y_{1}\right), x_{2}(t) \in S\left(y_{2}\right)$ such that

$$
d_{H}\left(S\left(y_{1}\right), S\left(y_{2}\right)\right)=\left\|x_{1}(t)-x_{2}(t)\right\|_{C(I, H)} .
$$

Note that

$$
x_{i}(t)=\int_{0}^{t} \dot{x}_{i}(s) d s+\tau\left(y_{i}(t)\right),
$$


for $i=1,2$. By (3.11), one has

$$
\begin{aligned}
& x_{1}(t)-x_{2}(t)+\int_{0}^{t}\left(B\left(s, x_{1}(s)\right)-B\left(s, x_{2}(s)\right)\right) d s+\int_{0}^{t}\left(D x_{1}(s)-D x_{2}(s)\right) d s \\
& \quad=\tau\left(y_{1}\right)-\tau\left(y_{2}\right)
\end{aligned}
$$

for every $y_{1}, y_{2} \in C(I, H)$. Taking the inner product over (3.15) with $x_{1}(t)-x_{2}(t)$, by (H1)(ii) and $(\mathrm{H} 2)_{1}(\mathrm{i})$, then

$$
\left\langle x_{1}(t)-x_{2}(t), x_{1}(t)-x_{2}(t)\right\rangle \leq\left\langle\tau\left(y_{1}(t)\right)-\tau\left(y_{2}(t)\right), x_{1}(t)-x_{2}(t)\right\rangle \text {. }
$$

It follows that

$$
\left\|x_{1}-x_{2}\right\|_{C(I, H)} \leq\left\|\tau\left(y_{1}\right)-\tau\left(y_{2}\right)\right\|_{H} .
$$

By $(\mathrm{H} 2)_{1}(\mathrm{ii})$, we have

$$
\left\|x_{1}-x_{2}\right\|_{C(I, H)} \leq \mathcal{L}\left\|y_{1}-y_{2}\right\|_{C(I, H)}
$$

From (3.14), one has

$$
d_{H}\left(S\left(y_{1}\right), S\left(y_{2}\right)\right) \leq \mathcal{L}\left\|y_{1}-y_{2}\right\|_{C(I, H)} .
$$

Since $\mathcal{L} \in[0,1)$, from Nadler's fixed-point theorem [23] and Theorem 1 in [24], we know that $S=\{y: y \in S(y)\}$ is an absolute retract in $C(I, H)$, which shows that $S$ is path connected in $C(I, H)$. The proof is completed.

Remark 3.2 In [25], it is proved that the set of extremal solutions of a differential inclusion in $\mathbb{R}^{N}$ is path connected based on the Baire category method.

\section{Examples}

To reveal the effectiveness of the previous results, we introduce two examples. Our examples are inspired directly by the work of [1], Examples, and [26], but here our boundary value conditions more general. Furthermore, we obtain the results which are new on the structural properties of solution set for the following examples. Let $\Omega \subset \mathbb{R}^{N}$ be a bounded domain with smooth boundary $\partial \Omega, I=[0, T], 0<T<\infty$. Let $q$ be the conjugate of $p$ and $p>1$. Let $\Delta=\sum_{k=1}^{N} \frac{\partial^{2}}{\partial x_{k}^{2}}$. We first consider a nonlinear evolution equation with a discontinuous right-hand side of the form

$$
\begin{aligned}
& \frac{\partial u(t, x)}{\partial t}-\operatorname{div}\left(|\nabla u(t, x)|^{p-2} \nabla u(t, x)\right)+|u(t, x)|^{p-2} u(t, x)-\Delta u(t, x) \\
& \quad=g(t, x, u(t, x)) \quad \text { for }(t, x) \in I \times \Omega, \\
& u(t, x)=0 \quad \text { for }(t, x) \in I \times \partial \Omega, \\
& u(0, x)=\frac{\theta_{1}}{T} \int_{0}^{T} u(t, x) d t+\theta_{2} u(T, x) \quad \text { for } x \in \Omega,
\end{aligned}
$$


where $p>1, \theta_{1}, \theta_{2} \in[-1,1]$, and $\left|\theta_{1}\right|+\left|\theta_{2}\right| \leq 1$. In general, the function $g(t, x, \cdot)$ is discontinuous. In order to obtain the nonemptiness of the solution set for (4.1), we change to a multivalued problem by, generally speaking, cramming the gaps at the discontinuous points of $g(t, x, \cdot)$. To this end, we introduce the functions $g_{1}(t, x, u)$ and $g_{2}(t, x, u)$ defined by

$$
g_{1}(t, x, u)=\liminf _{\xi \rightarrow u} g(t, x, \xi) \quad \text { and } \quad g_{2}(t, x, u)=\limsup _{\xi \rightarrow u} g(t, x, \xi)
$$

For all $(t, x) \in I \times \Omega$, we can define the set-valued function $F(t, x, u)$ by

$$
F(t, x, u)=\left[g_{1}(t, x, u), g_{2}(t, x, u)\right]=\left\{v \in \mathbb{R}: g_{1}(t, x, u) \leq v \leq g_{2}(t, x, u)\right\}
$$

Next, corresponding to (4.1), we consider the following evolution inclusion:

$$
\begin{aligned}
& \frac{\partial u(t, x)}{\partial t}-\operatorname{div}\left(|\nabla u(t, x)|^{p-2} \nabla u(t, x)\right)+|u(t, x)|^{p-2} u(t, x)-\Delta u(t, x) \\
& \quad \in F(t, x, u(t, x)) \quad \text { for }(t, x) \in I \times \Omega, \\
& u(t, x)=0 \quad \text { for }(t, x) \in I \times \partial \Omega, \\
& u(0, x)=\frac{\theta_{1}}{T} \int_{0}^{T} u(t, x) d t+\theta_{2} u(T, x) \quad \text { for } x \in \Omega .
\end{aligned}
$$

To get the topology structure of the solution set of (4.1), we need the following hypotheses on $g$.

(H5) $g: I \times \Omega \times \mathbb{R} \rightarrow \mathbb{R}$ is a function such that

(i) if $u: I \times \Omega \rightarrow \mathbb{R}$ is a measurable function, then so are $(t, x) \rightarrow g_{1}(t, x, u(t, x)), g_{2}(t, x, u(t, x))$

(ii) for almost all $(t, x) \in I \times \Omega$ and all $u \in \mathbb{R}$, there exist $a_{2}(t, x) \in L_{+}^{q}\left(I, L^{2}(\Omega)\right)$ and $\widehat{c}(x) \in L^{\infty}(\Omega)$ such that

$$
|g(t, x, u)| \leq a_{2}(t, x)+\widehat{c}(x)|u|^{k-1},
$$

with $1 \leq k<p$.

The evolution triple is, in this case, $H=L^{2}(\Omega), V=W_{0}^{1, p}(\Omega)$, and $V^{*}=W^{-1, q}(\Omega)$. Due to the Sobolev embedding theorem, we know that all embeddings are compact. Let us define the following operator on $W_{0}^{1, p}(\Omega)$ :

$$
\langle B(u)(t), v\rangle_{*}=\int_{\Omega}\left(|\nabla u|^{p-2} \nabla u \cdot \nabla v+|u|^{p-2} u v\right) d x .
$$

By the monotone property of $p$-Laplacian, it is easy to deduce that $B$ satisfies our hypothesis (H1). Also we define the operator $D$ by

$$
\langle D(u)(t), v\rangle_{*}=\int_{\Omega} \nabla u \cdot \nabla v d x .
$$

Evidently, (H2)(i) holds. Next, let $\mathcal{F}: I \times H \rightarrow P_{f c}(H)$ be defined by

$$
\mathcal{F}(t, u)=\left\{v \in L^{2}(\Omega): g_{1}(t, x, u) \leq v(x) \leq g_{2}(t, x, u)\right\} .
$$


Hypothesis (H5) shows that (H3) is satisfied. Since $g_{1}(t, x, \cdot)$ is lower semicontinuous, $g_{2}(t, x, \cdot)$ is upper semicontinuous, and so $F(t, x, \cdot)$ is upper semicontinuous (see [15], Example 2.8, p.371]). Finally, let $\tau: C\left(I, L^{2}(\Omega)\right) \rightarrow L^{2}(\Omega)$ be defined by $\tau(u)=\frac{\theta_{1}}{T} \int_{0}^{T} u(t, x) d t+$ $\theta_{2} u(T, x)$, we have

$$
\begin{aligned}
\left\|\tau\left(u_{1}\right)-\tau\left(u_{2}\right)\right\|_{L^{2}} \leq & \frac{\left|\theta_{1}\right|}{T}\left\|\int_{0}^{T} \max _{t \in I}\left|u_{1}(t, x)-u_{2}(t, x)\right| d t\right\|_{L^{2}} \\
& +\left|\theta_{2}\right| \max _{t \in I}\left\|u_{1}(t, x)-u_{2}(t, x)\right\|_{L^{2}} \\
\leq & \left\|u_{1}-u_{2}\right\|_{C\left(I, L^{2}(\Omega)\right)},
\end{aligned}
$$

for every $u_{1}, u_{2} \in C\left(I, L^{2}(\Omega)\right)$, which means $\tau$ satisfies our hypothesis (H2)(ii). Then we solve equivalently (4.1) as (3.1), with $B, D$, and $\mathcal{F}$ as above. Finally, we can use Theorem 3.1 on problem (4.1) and obtain the following consequence.

Theorem 4.1 If hypothesis (H5) is satisfied, then the solution set $S$ of (4.1) is nonempty and compact $R_{\delta}$ in $C\left(I, L^{2}(\Omega)\right)$. Moreover, it is compact and connected in $C\left(I, L^{2}(\Omega)\right)$.

Second, we offer an example of a quasilinear control system, with a prior feedback. Let $I=[0, T]$ where $0<T<\infty$, and $\Omega \subseteq \mathbb{R}^{N}$ a bounded domain with $C^{2}$-boundary $\partial \Omega$. Let $D_{k}=\frac{\partial}{\partial x_{k}}, D=\left(D_{1}, \ldots, D_{N}\right), \Delta=\sum_{k=1}^{N} \frac{\partial^{2}}{\partial x_{k}^{2}}$. We examine the following control system:

$$
\begin{aligned}
& \frac{\partial u(t, x)}{\partial t}-\sum_{k=1}^{N} D_{k} B_{k}(t, x, u(t, x), D u(t, x))-\Delta u(t, x) \\
& =f(t, x, u(t, x)) v(t, x) \quad \text { for }(t, x) \in I \times \Omega, \\
& u(t, x)=0 \quad \text { for }(t, x) \in I \times \partial \Omega, \\
& u(0, x)=\frac{1}{2 T} \int_{0}^{T} u(t, x) d t \quad \text { for } x \in \Omega, \\
& v(t, x) \in U(t, x, u(t, x)) \quad \text { for }(t, x) \in I \times \Omega .
\end{aligned}
$$

The following hypotheses on (4.3) are needed:

(H6) $B_{k}(k=1,2, \ldots, N): I \times \Omega \times \mathbb{R} \times \mathbb{R}^{N} \rightarrow \mathbb{R}$ are functions such that

(i) $(t, x) \rightarrow B_{k}(t, x, u, \xi)$ is measurable on $I \times \Omega$ for every $(u, \xi) \in \mathbb{R} \times \mathbb{R}^{N}$, $(u, \xi) \rightarrow B_{k}(t, x, u, \xi)$ is continuous on $\mathbb{R} \times \mathbb{R}^{N}$ for almost all $(t, x) \in I \times \Omega$;

(ii) $\left|B_{k}(t, x, u, \xi)\right| \leq \hat{\alpha}_{1}(t, x)+\hat{c}_{1}(x)(|u|+|\xi|)$ with a function $\hat{\alpha}_{1} \in L_{+}^{2}\left(I, L^{2}(\Omega)\right)$ and $\hat{c}_{1}(z) \in L^{\infty}(\Omega)$ for almost all $t \in I$;

(iii) $\sum_{k=1}^{N}\left(B_{k}(t, x, u, \xi)-B_{k}\left(t, x, u, \xi^{\prime}\right)\right)\left(\xi_{k}-\xi_{k}^{\prime}\right) \geq\left|\xi-\xi^{\prime}\right|^{2}$ for almost all $t \in I$;

(iv) $B_{k}(t, x, 0,0)=0$ for all $(t, x) \in I \times \Omega$.

(H7) The functions $f: I \times \Omega \times \mathbb{R} \rightarrow \mathbb{R}$ satisfies

(i) for all $u \in \mathbb{R},(t, x) \rightarrow f(t, x, u)$ is measurable;

(ii) for all $(t, x) \in I \times \Omega, u \rightarrow f(t, x, u)$ is continuous;

(iii) for almost all $(t, x) \in I \times \Omega$ and all $u \in \mathbb{R}$, we have

$$
|f(t, x, u)| \leq \eta_{1}(t, x)+\eta_{2}(x)|u|
$$

where $\eta_{1} \in L^{2}\left(I, L^{2}(\Omega)\right), \eta_{2} \in L_{+}^{\infty}(\Omega)$. 
(H8) $U: I \times \Omega \times \mathbb{R} \rightarrow P_{f}(\mathbb{R})$ is a multifunction such that

(i) for all $u \in \mathbb{R},(t, x) \rightarrow U(t, x, u)$ is measurable;

(ii) for almost all $(t, x) \in I \times \Omega$ and all $u_{1}, u_{2} \in L^{2}(\Omega)$,

$$
d_{H}\left(U\left(t, x, u_{1}\right), U\left(t, x, u_{2}\right)\right) \leq z(t)\left\|u_{1}-u_{2}\right\|_{L^{2}} \text { with } z(t) \in L_{+}^{1}(I) ;
$$

(iii) for almost all $(t, x) \in I \times \Omega$ and all $u \in \mathbb{R},|U(t, x, u)| \leq M$, where $M>0$.

Let $\left(V, H, V^{*}\right)$ be an evolution triple with compact embeddings where $V=H_{0}^{1}(\Omega), H=$ $L^{2}(\Omega), V^{*}=H^{-1}(\Omega)$. Let $B: I \times V \rightarrow V^{*}, D: V \rightarrow V^{*}$ be the operators defined by

$$
\begin{aligned}
& \langle B(t, u), w\rangle=\int_{\Omega} \sum_{k=1}^{N} B_{k}(t, x, u(t, x), D u(t, x)) D_{k} w(x) d x, \\
& \langle D u, w\rangle=\int_{\Omega} \sum_{k=1}^{N} D_{k} u(t, x) D_{k} w(x) d x
\end{aligned}
$$

for all $w \in H_{0}^{1}(\Omega)$. Let $\tau: C\left(I, L^{2}(\Omega)\right) \rightarrow L^{2}(\Omega)$ be defined by $\tau(u)=\frac{1}{2 T} \int_{0}^{T} u(t, x) d t$. Evidently, by hypotheses (H6), it is forthright to verify that $B, D, \tau$ satisfy hypotheses (H1), $(\mathrm{H} 2)_{1}$. Also, let $\mathcal{F}: I \times L^{2}(\Omega) \rightarrow P_{f}\left(L^{2}(\Omega)\right)$ be defined by

$$
\mathcal{F}(t, u(t))=\left\{w \in L^{2}(\Omega): w(t, x)=g(t, x, u(t, x)) v(x), v(x) \in U(t, x, u(t, x)), \text { for } x \in \Omega\right\} .
$$

According to hypotheses (H7) and (H8), it is easy to verify that $\mathcal{F}$ satisfies hypotheses (H4).

Convert problem (4.3) to the following equivalent form:

$$
\begin{aligned}
& \frac{\partial u}{\partial t}+B(t, u(t))+D u(t) \in \mathcal{F}(t, u(t)) \quad \text { for } t \in I, \\
& u(0)=\tau(u) .
\end{aligned}
$$

By applying Theorem 3.2 on problem (4.4), we obtain the following theorem.

Theorem 4.2 If hypotheses (H6)-(H8) are satisfied, then the solution set $S$ of (4.3) is nonempty and path connected in $C\left(I, L^{2}(\Omega)\right)$.

\section{Competing interests}

The authors declare that they have no competing interests.

\section{Authors' contributions}

YC carried out the main part of this manuscript. BN participated in the discussion and corrected the main theorem. CL provided all examples for our results. All authors read and approved the final manuscript.

\section{Author details}

'Department of Mathematics, Bohai University, Jinzhou, 121012, P.R. China. ${ }^{2}$ The Center of Teaching Reform and Evaluation, Bohai University, Jinzhou, 121012, P.R. China.

\section{Acknowledgements}

This work is partially supported by National Natural Science Foundation of China (No.11401042, 10902125, 61304054) and the Program for Liaoning Provincial Excellent Talents in University, China (LJQ2014122). The authors would like to express their sincere appreciation to the reviewer for his/her helpful comments in improving the presentation and quality of the paper.

Received: 15 October 2015 Accepted: 12 January 2016 Published online: 28 January 2016 


\section{References}

1. Zhang, J, Cheng, Y, Yuan, C, Cong, F: Properties of the solutions set for a class of nonlinear evolution inclusions with nonlocal conditions. Bound. Value Probl. 2013, 15 (2013)

2. Aizicovici, S, Lee, H: Nonlinear nonlocal Cauchy problems in Banach spaces. Appl. Math. Lett. 18, $401-407$ (2005)

3. Aizicovici, S, McKibben, M: Existence results for a class of abstract nonlocal Cauchy problems. Nonlinear Anal. 39, 649-668 (2000)

4. Aizicovici, S, Staicu, V: Multivalued evolution equations with nonlocal initial conditions in Banach spaces. Nonlinear Differ. Equ. Appl. 14, 361-376 (2007)

5. García-Falset, J: Existence results and asymptotic behaviour for nonlocal abstract Cauchy problems. J. Math. Anal. Appl. 338, 639-652 (2008)

6. García-Falset, J, Reich, S: Integral solutions to a class of nonlocal evolution equations. Commun. Contemp. Math. 12 1031-1054 (2010)

7. Paicu, A, Vrabie, II: A class of nonlinear evolution equations subjected to nonlocal initial conditions. Nonlinear Anal. 72, 4091-4100 (2010)

8. Himmelberg, C, Van Vleck, F: A note on the solution sets of differential inclusions. Rocky Mt. J. Math. 12, 621-625 (1982)

9. DeBlasi, FS, Myjak, J: On the solution set for differential inclusions. Bull. Pol. Acad. Sci., Math. 33, 17-23 (1985)

10. Papageorgiou, NS, Shahzad, N: Properties of the solution set of nonlinear evolution inclusion. Appl. Math. Optim. 36 1-20 (1997)

11. Andres, J, Pavlǎcková, M: Topological structure of solution sets to asymptotic boundary value problems. J. Differ. Equ. 248, 127-150 (2010)

12. Chen, DH, Wang, RN, Zhou, Y: Nonlinear evolution inclusions: topological characterizations of solution sets and applications. J. Funct. Anal. 265(9), 2039-2073 (2013)

13. Papageorgiou, NS, Yannakakis, N: Second order nonlinear evolution inclusions II: structure of the solution set. Acta Math. Sci. 22(1), 195-206 (2006)

14. Mahmudov, EN: Optimization of Cauchy problem for partial differential inclusions of parabolic type. J. Dyn. Control Syst. 20, 176-180 (2014)

15. Hu, S, Papageorgiou, NS: Handbook of Multivalued Analysis. Vol. I. Theory. Kluwer Academic, Dordrecht (1997)

16. Hu, S, Papageorgiou, NS: Handbook of Multivalued Analysis. Vol. II. Applications. Kluwer Academic, Dordrecht (2000)

17. Zeidler, E: Nonlinear Functional Analysis and Its Applications. Vol. II. Springer, Berlin (1990)

18. DeBlasi, FS, Myjak, J: On continuous approximations for multifunctions. Pac. J. Math. 123, 9-31 (1986)

19. Papageorgiou, NS: On measurable multifunctions with applications to random multivalued equations. Math. Jpn. 32, 437-464 (1987)

20. Papageorgiou, NS: Existence of solutions for second-order evolution inclusions. J. Appl. Math. Stoch. Anal. 7, 525-535 (1994)

21. Brezis, H: Operateurs Maximaux Monotones. North-Holland, Amsterdam (1973)

22. Hyman, DM: On decreasing sequences of compact absolute retracts. Fundam. Math. 64, 91-97 (1969)

23. Nadler, S: Multivalued contraction mappings. Pac. J. Math. 30, 475-488 (1969)

24. Bressan, A, Cellina, A, Fryszkowski, A: A class of absolute retracts in spaces of integrable functions. Proc. Am. Math. Soc. 112, 413-418 (1991)

25. DeBlasi, FS, Pianigiani, G: Topological properties of nonconvex differential inclusions. Nonlinear Anal. TMA 20, 871-894 (1993)

26. Xue, X, Cheng, Y: Existence of periodic solutions of nonlinear evolution inclusions in Banach spaces. Nonlinear Anal., Real World Appl. 11, 459-471 (2010)

\section{Submit your manuscript to a SpringerOpen ${ }^{\circ}$ journal and benefit from:}

$\checkmark$ Convenient online submission

- Rigorous peer review

Immediate publication on acceptance

- Open access: articles freely available online

- High visibility within the field

- Retaining the copyright to your article 